\title{
Germinação de sementes de três espécies de Fabaceae típicas de floresta estacional decidual
}

\author{
Daniel Meira Arruda ${ }^{1}$, Diego Oliveira Brandão², Maria das Dores Magalhães Veloso ${ }^{3}$, Yule Roberta Ferreira Nunes ${ }^{3^{*}}$ \\ ${ }^{1}$ Universidade Federal de Viçosa, Departamento de Biologia Vegetal, Av P.H. Rolfs, s/nº, Centro, CEP 36571-000, Viçosa, MG, Brasil \\ ${ }^{2}$ Amazônia Socioambiental, Incubadora de Empresa - Instituto Nacional de Pesquisas da Amazônia. Av André Araújo, 2.936, CEP 69067-375, Manaus, AM, Brasil \\ ${ }^{3}$ Universidade Estadual de Montes Claros, Departamento de Biologia Geral, Campus Universitário Darci Ribeiro S/N, Vila Mauricéia, CEP 39401-089, Montes \\ Claros, MG, Brasil
}

"Autor correspondente:

yule.nunes@unimontes.br

Termos para indexação:

Dormência de sementes

Floresta decídua

Mata seca

Predação de sementes

Propagação

Restauração

\section{Index terms:}

Seed dormancy

Deciduous forest

Tropical dry forest

Seed predation

Propagation

Restoration

Histórico do artigo:

Recebido em 10/03/2014

Aprovado em 01/06/2015

Publicado em 30/06/2015

doi: $10.4336 / 2015$.pfb.35.82.672
Resumo - O presente estudo avalia a germinação de sementes de Anadenanthera colubrina, Acacia polyphylla e Bauhinia cheilantha, espécies típicas de florestas decíduas, submetidas a tratamentos pré-germinativos e ataque de insetos nativos. As sementes de cada espécie foram agrupadas em: sementes escarificadas com lixa, sementes imergidas em água aquecida a $70^{\circ} \mathrm{C}$, sementes com sinais de ataque por insetos e o grupo controle. A maior proporção de sementes germinadas ocorreu na primeira semana de incubação, com pico de germinação variando do primeiro ao terceiro dia. Independente do grupo avaliado, $A$. polyphylla e $B$. cheilantha apresentaram elevada taxa de germinação $(>90 \%)$, sendo reduzida apenas quando as sementes apresentaram ataque por insetos $(<25 \%)$. O grupo de escarificação mecânica em $\mathrm{A}$. polyphylla apresentou máxima germinação $(100 \%)$ e aumentou a velocidade da germinação. Em A. colubrina, não houve diferença entre os grupos e a taxa de germinação foi inferior $(<50 \%)$, a qual foi atribuída à infestação por fungos, comumente relatada em experimentos com a espécie, independente dos procedimentos usuais de higienização. Salvo efeito de infestação por fungos em A. colubrina, as espécies avaliadas mostraramse independentes de tratamentos pré-germinativos para obter uma elevada taxa de germinação.

\section{Seed germination of three species of Fabaceae typical of seasonally dry forest}

\begin{abstract}
This study evaluates seeds germination of Anadenanthera colubrina, Acacia polyphylla and Bauhinia cheilantha, typical species of deciduous forests. Seeds were submitted to pre-germination treatments and attack of native insects. The seeds of each species were grouped in: seeds scarified with sandpaper; seeds immersed in water heated to $70^{\circ} \mathrm{C}$, seeds with signs of attack by herbivore insects and the control group. The largest proportion of germinated seeds occurred in the first week of incubation and germination peak, ranged from first to third day. All groups of A. polyphylla and B.cheilantha showed high germination rate $(>90 \%)$, being reduced only when seeds were attacked by insects $(<25 \%)$. Mechanic scarification was efficient in A. polyphylla by enhancing germination to maximum $(100 \%)$ and accelerating germination. A. colubrina showed no difference among groups, and germination rate was lower $(<50 \%)$, which was attributed to infestation by fungi, commonly reported in this species and apparently independent of usual hygiene procedures. Finally, except the fungi infestation in A. colubrina, evaluated species were independent of pre-germination treatment to obtain a high rate of germination.
\end{abstract}




\section{Introdução}

Agerminação consiste na retomada do desenvolvimento embrionário da semente, resultado de uma série de mudanças enzimáticas e bioquímicas que promove a exposição da radícula (Labouriau, 1983). Entretanto, quando a semente não germina em condições ambientais favoráveis, geralmente é um sinal de imaturidade embrionária ou de alguma restrição física imposta pelo próprio propágulo. Essa limitação é conhecida como dormência e sua origem acontece na fase de maturação da semente de algumas espécies (Bewley \& Black, 1985).

Sementes de espécies florestais geralmente apresentam algum tipo de dormência, o que pode ser um obstáculo para o manejo florestal (Nunes et al., 2006), pois aumenta a variabilidade dos lotes germinados e interfere na avaliação da qualidade da semente (Popinigis, 1985). No entanto, a dormência pode ser superada através de métodos abrasivos impostos à semente, conhecidos como escarificação (Cardoso, 2009). Na natureza, uma forma comum de escarificação é a ação dos herbívoros nas sementes (Copeland \& McDonald, 2001), uma vez que essas são potenciais fontes de alimento para aqueles organismos (Janzen, 1975; Hulme, 1998). Desse modo, o ataque de herbívoros pode resultar em um orifício no tegumento da semente que viabiliza a entrada de recursos, como água, luz e oxigênio, favorecendo a germinação, ou pode lesionar o embrião da semente causando sua morte (Copeland \& McDonald, 2001).

Em laboratório, técnicas pré-germinativas são utilizadas para potencializar a germinação. A exposição da semente em água aquecida é uma técnica que busca acelerar a atividade enzimática da mesma (Zaidan \& Barbedo, 2004) ou mesmo diminuir a rigidez do seu tegumento (Fachinello et al., 2005). De forma semelhante, a remoção de uma parte do tegumento da semente pode promover a germinação, por facilitar a entrada de recursos (Borges \& Rena, 1993; MeloPinna et al., 1999; Luz \& Nunes 2013). Esses recursos, combinados ou não com a temperatura (Araujo Neto et al., 2003; 2005), causam a reativação do metabolismo respiratório, seguido da ativação enzimática, consumo das reservas cotiledonares e rompimento dos envoltórios da semente (Labouriau, 1983; Corte et al., 2006).

Os estudos de germinação de sementes são importantes por contribuírem para a propagação de mudas (Nunes et al., 2006), principalmente quando se trata de ecossistema com elevado grau de ameaça (Janzen, 1988) e de poucos esforços de pesquisa (Sanchez-Azofeifa et al., 2005), como são as florestas estacionais deciduais.

Segundo Oliveira-Filho et al. (2006), Fabaceae é a família mais representativa das florestas decíduas, e o estudo de suas espécies se faz de grande importância para conservação dessas florestas. Embora adaptada para suportar o clima seco, as sementes de Fabaceae geralmente apresentam alguma dormência tegumentar (Melo-Pinna et al., 1999; Zaidan \& Barbedo, 2004; Luz \& Nunes, 2013), o que representa um obstáculo na produção de mudas para iniciativas de restauração. No entanto, nem todas as sementes de Fabaceae apresentam tal característica (Lorenzi, 1992, Barbosa, 2003; Carvalho, 2003, 2008; Matheus \& Lopes, 2007; Pereira, 2011), principalmente espécies florestais de Caatinga, as quais apresentam rápida germinação (Barbosa, 2003).

Nesse contexto, o presente estudo teve o objetivo de analisar o potencial germinativo de três espécies de Fabaceae típicas de floresta decídua, Anadenanthera colubrina (Vell.) Brenan, Acacia polyphylla DC. e Bauhinia cheilantha (Bong.) Steud., sob o efeito de tratamentos pré-germinativos e ataque de insetos nativos.

\section{Material e métodos}

O estudo foi conduzido no Laboratório de Ecologia e Propagação Vegetal da Universidade Estadual de Montes Claros. Os frutos foram coletados no final da estação seca de 2006 (novembro) e início da estação seca de 2007 (maio), e postos para germinar sem período de armazenamento. Os frutos foram oriundos de matrizes presentes em um fragmento de Floresta Estacional Decidual, localizado em Montes Claros, MG (16 $38^{\circ}$ '53" S e 43 53'30" W). O clima da região é do tipo Aw, com temperatura média anual de $22,4{ }^{\circ} \mathrm{C}$ e precipitação média anual de $1.100 \mathrm{~mm}$, sendo seis meses com precipitação inferior a $60 \mathrm{~mm}$ (Antunes, 1994).

Após a secagem natural dos frutos em laboratório, as sementes foram extraídas, separadas em lotes homogêneos, sendo estes submetidos a dois tratamentos pré-germinativos: escarificação mecânica, na qual o tegumento da semente foi lixado cerca de $2 \mathrm{~mm}$ com uma lixa ${ }^{\circ} 80$ no lado oposto ao hilo, até o aparecimento do cotilédone (Nunes et al., 2006); e imersão em água aquecida, na qual as sementes foram imersas em água a $70{ }^{\circ} \mathrm{C}$ por $5 \mathrm{~min}$. Além do grupo controle, outro grupo de sementes foi separado e posto para germinar, sendo 
este composto pelas sementes que apresentavam dano no tegumento causado por insetos (salvo em Anadenanthera colubrina, por não apresentar essa associação), totalizando quatro grupos. Os insetos encontrados no interior das sementes de Bauhinia cheilantha e Acacia polyphylla representavam quatro espécies em vários estágios larvais, sendo duas espécies da ordem Braconidae, uma de Bruchidae e uma de Perilampidae.

As sementes foram colocadas em gerbox esterilizados, sobre papel filtro umedecido com água destilada. Em cada tratamento, foram utilizados 14 gerbox com 15 sementes $(\mathrm{n}=210)$ para $A$. colubrina, 10 gerbox com 20 sementes $(\mathrm{n}=200)$ para $A$. polyphylla, e 10 gerbox com 10 sementes $(n=100)$ para $B$. cheilantha. Os gerbox foram organizados de forma aleatória e acondicionados em germinador (FANEM modelo $347 \mathrm{CDG}$ ) programado para que a temperatura e luz se alterassem a cada $12 \mathrm{~h}$, sendo $30^{\circ} \mathrm{C}$ com luz e $20^{\circ} \mathrm{C}$ sem luz. As sementes foram avaliadas diariamente durante 30 dias e umedecidas sempre que necessário. Foi considerada germinada a semente que havia emitido raiz primária, conforme critério definido por Labouriau (1983). Além da porcentagem de germinação de sementes de cada grupo, também foi considerado o índice de velocidade de germinação (IVG), adaptado da fórmula de Maguire (1962). A adaptação consistiu em substituir a contagem das sementes germinadas pela porcentagem, tornando possível a comparação entre as espécies, uma vez que diferentes quantidades de sementes foram utilizadas. Dessa forma, a fórmula utilizada foi:

$I V G=\left(P_{1} / T_{1}\right)+\left(P_{2} / T_{2}\right)+\ldots+\left(P_{n} / T_{n}\right)$

Onde $\mathrm{P}=$ porcentagem de sementes germinadas na primeira, segunda, n-ésima avaliação; e $\mathrm{T}=$ tempo decorrido (dias) da primeira; segunda; n-ésima avaliação.

Para verificar a diferença entre os grupos, os dados obtidos da germinação foram submetidos à análise de variância. Para os dados com distribuição normal e com amostras homogêneas, foi executada ANOVA seguida do teste Tukey a 5\% de probabilidade. Quando os dados não atendiam os pressupostos para a análise paramétrica, foi executada a análise Kruskall-Wallis seguida do teste Mann-Whitney a 5\% de probabilidade. As análises foram feitas no software estatístico PAST 2.15 (Hammer et al., 2001).

\section{Resultados e discussão}

A maior proporção de sementes germinadas das diferentes espécies avaliadas ocorreu nos três primeiros dias. Salvo os grupos das sementes atacadas por insetos (referentes a Acacia polyphylla e Bauhinia cheilantha), os grupos de todas as espécies tiveram elevada velocidade de germinação (Tabela 1). Essa rápida resposta à germinação, entre dois e cinco dias, é descrita por Barbosa (2003) para diferentes espécies do semiárido. O pico de germinação variou entre o primeiro e o terceiro dia de acordo com a espécie e o grupo. Acacia polyphylla obteve pico de germinação no terceiro dia, em todos os grupos (Figura 1A), enquanto Anadenanthera colubrina (Figura 1B) e B. cheilantha (Figura 1C) tiveram maior variação entre os grupos. Anadenanthera colubrina apresentou o pico de germinação no primeiro dia para as sementes lixadas e no segundo dia para os demais grupos, enquanto $B$. cheilantha obteve o pico no segundo e terceiro dia para as sementes lixadas e no terceiro dia, para os demais grupos. De modo geral, a maior proporção de sementes germinadas (>90\%) ocorreu durante a primeira semana de incubação, o que demonstra uma alta sincronia de germinação entre as sementes das diferentes espécies.

Tabela 1. Índice de velocidade de germinação das espécies avaliadas nos diferentes grupos.

\begin{tabular}{lcccc}
\hline & Controle & Água $70{ }^{\circ} \mathrm{C}$ & $\begin{array}{c}\text { Escarificadas } \\
\text { com lixa }\end{array}$ & $\begin{array}{c}\text { Atacadas } \\
\text { por } \\
\text { insetos }\end{array}$ \\
\hline A. colubrina & 26,15 & 23,21 & 31,19 & - \\
A. polyphyla & 26,17 & 27,09 & 38,43 & 6,72 \\
B. cheilantha & 31,22 & 37,35 & 33,52 & 4,42 \\
\hline
\end{tabular}

A rápida germinação encontrada no presente estudo é decorrente dos primeiros dias de umedecimento, indicando não haver grandes barreiras para sua hidratação, como demonstrado por Barbosa (2003) e Matheus \& Lopes (2007) para outras espécies de Fabaceae. Em ambientes sazonais, espécies que dispersam suas sementes na estação seca têm rápida resposta germinativa no início da estação úmida (Garwood, 1983; Nunes et al., 2008). O início da estação úmida é um momento favorável para a germinação rápida, pois as plântulas teriam mais tempo para crescer e tentar se estabelecer na comunidade (Augspurger, 1990; Felfili et al., 1999). Por outro lado, quando a germinação ocorre em períodos desfavoráveis, como resultado de chuvas esporádicas, a plântula pode não sobreviver ao déficit hídrico imposto pela estação seca (Barbosa, 2003; Zaidan \& Barbedo, 2004).

Pesq. flor. bras., Colombo, v. 35, n. 82, p. 135-142, abr./jun. 2015 
Os grupos não diferiram em $A$. colubrina $(\mathrm{F}=$ $0,29 ; \mathrm{gl}=2 ; \mathrm{p}=0,75)$. O percentual de germinação de cada grupo não ultrapassou $50 \%$ (controle: $46,7 \pm$ 14,3; lixada: $49,5 \pm 13,2$ e água $\left.70{ }^{\circ} \mathrm{C}: 45,2 \pm 16,0\right)$, diferente de outros estudos que atingiram germinação superior a 75\% (Garcia et al., 2004; Melo et al., 2005). No entanto, a semelhança entre os grupos dessa espécie indica ausência de dormência (Lorenzi, 1992; Barbosa, 2003; Pereira, 2011). Possivelmente, a baixa germinação das sementes de $A$. colubrina está relacionada à quiescência, necessitando de condições mais favoráveis de luz e clima (Figlioli et al., 2009), ou foi devido à imprevista contaminação por fungos a partir do segundo dia do experimento (Figura 1B). Segundo Borges \& Rena (1993), fungos podem prejudicar a entrada de oxigênio, afetando negativamente os processos metabólicos das sementes (Borges \& Rena, 1993).
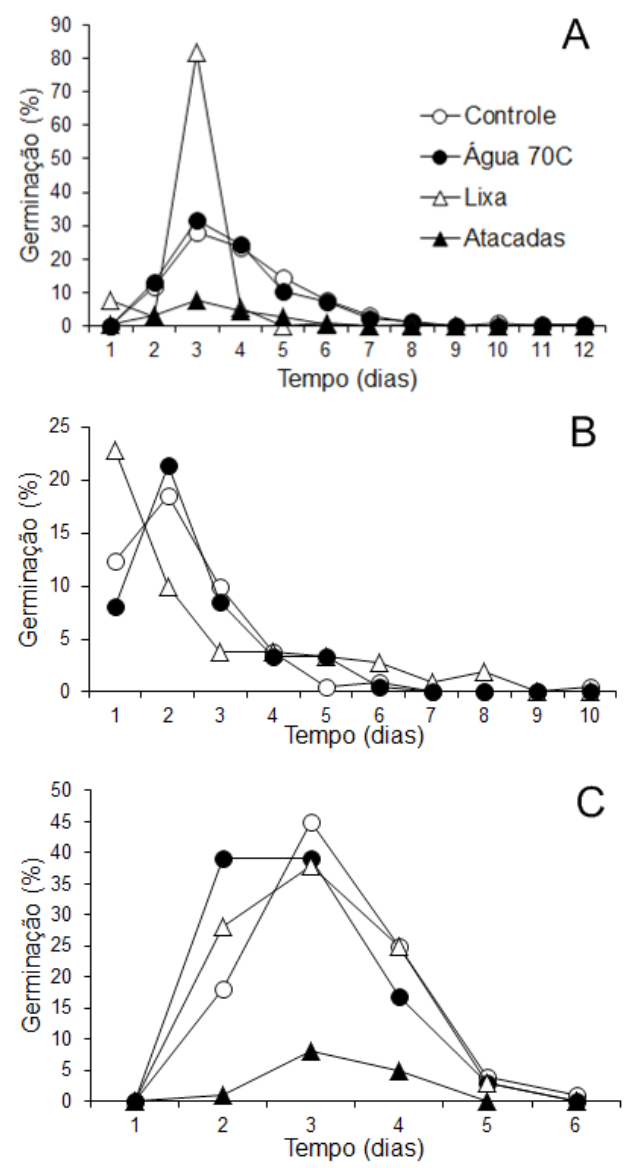

Figura 1. Porcentagem de sementes germinadas nos dias de incubação. (A) Acacia polyphylla, (B) Anadenanthera colubrina e (C) Bauhinia cheilantha correspondentes aos grupos de sementes lixadas, sementes emergidas em água aquecida a $70{ }^{\circ} \mathrm{C}$, sementes atacadas por insetos e o grupo controle.

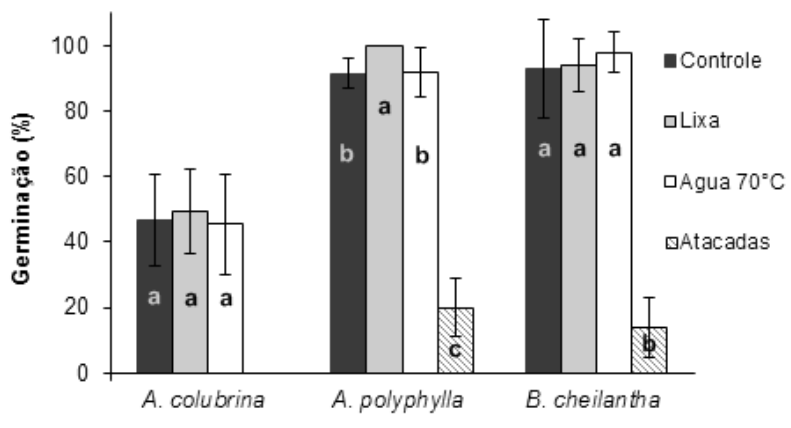

Figura 2. Porcentagem média e desvio padrão da germinação das sementes de três espécies de Fabaceae representadas pelos grupos de sementes lixadas, sementes emergidas em água aquecida a $70{ }^{\circ} \mathrm{C}$, sementes atacadas por insetos e o grupo controle.

A contaminação por fungos em experimentos com A. colubrina é recorrente e não está associada com os procedimentos usuais de esterilização. Em estudo de germinação de sementes de $A$. colubrina oriundas de duas localidades, em que foram aplicados os mesmos procedimentos de esterilização utilizados no presente trabalho, Rodrigues et al. (2007) observaram que apenas um dos lotes ficou contaminado por fungos, acarretando em menor valor de germinação. Segundo Dhingra et al. (2002), a contaminação por fungo na espécie começa na formação da semente, sendo potencializada durante o seu processo de maturação.

Diferente de $A$. colubrina, as demais espécies tiveram altas taxas de germinação, com diferença entre os grupos. Acacia polyphylla variou entre os grupos $\left(\mathrm{X}^{2}=29,17\right.$; $\mathrm{gl}=3 ; \mathrm{p}<0,01)$. As sementes lixadas apresentaram germinação total, significativamente diferente dos demais grupos $(\mathrm{p}<0,01)$, e maior velocidade de germinação. O grupo com água aquecida $(92,0 \% \pm 7,9)$ foi semelhante apenas ao controle $(91,5 \% \pm 4,7)(\mathrm{p}=$ $0,64)$. As sementes atacadas obtiveram a menor taxa de germinação 20,0\% $( \pm 6,2)$, significativamente diferente dos demais grupos $(\mathrm{p}<0,01)$ (Figura 2). Devido a maior germinação apresentada pelas sementes lixadas, a espécie indicou uma dormência tegumentar. No entanto, a espécie pode obter elevadas taxas de germinação sem necessidade de tratamentos pré-germinativos (Lorenzi, 1992; Carvalho, 2008).

Bauhinia cheilantha também apresentou diferença significativa entre os grupos $(\mathrm{F}=127,3 ; \mathrm{gl}=3 ; \mathrm{p}<$ $0,01)$, sendo as sementes atacadas o grupo com menor percentual de germinação $(14,0 \% \pm 12,6)(p<0,01)$. Os 
demais grupos foram semelhantes $(\mathrm{p}>0,05)$ e tiveram elevadas porcentagens de germinação, com 93,0\% ( \pm $15,7)$ no grupo controle, $98,0 \%( \pm 6,3 \%)$ no grupo com água aquecida e $94,0 \%( \pm 8,4 \%)$ no grupo com as sementes lixadas, sem grandes diferenças na velocidade de germinação. Segundo Barbosa (2003), as sementes de $B$. cheilantha apresentam dormência tegumentar, necessitando de escarificação mecânica para germinar. No entanto, desconsideramos essa afirmação, devido à semelhança estatística entre os grupos com tratamento pré-germinativo e o controle.

Segundo Barbosa (2003), as sementes das espécies do semiárido geralmente apresentam percentual de germinação próximo de $90 \%$, corroborando com os resultados encontrados para $A$. polyphylla e $B$. cheilantha. Entretanto, algumas espécies necessitam que suas sementes sejam submetidas a temperaturas elevadas para que a germinação ocorra (Rodrigues et al., 1990; Nunes et al., 2006). Outras espécies podem perder a viabilidade com o aumento da temperatura (Givelberg et al., 1984; Oliveira et al., 2008). No entanto, os resultados do presente estudo indicam que as espécies avaliadas não necessitam de tratamentos pré-germinativos e não são inviabilizadas por eles, obtendo percentual elevado de germinação, salvo no grupo das sementes atacadas por insetos (Figura 2). Mas, no caso de A. polyphylla, a germinação pode ser potencializada ao máximo quando submetida à escarificação mecânica, além de aumentar a velocidade da germinação. Tal tratamento prégerminativo favorece a produção de mudas, reduzindo a variação do lote de sementes. Elevadas taxas de germinação em A. polyphylla também foi encontrada por Araújo Neto et al. (2003; 2005), ao tratar as sementes com diferentes intensidades de luz, temperatura e tempo de armazenamento, indicando ausência de dormência fisiológica (Carvalho et al., 2006; Silva et al., 2007; Lima et al., 2008).

A escarificação mecânica geralmente aumenta a taxa de germinação das sementes com tegumentos duros (Luz \& Nunes, 2013), como em outras espécies do gênero Bauhinia, B. variegata e B. forficata (Lopes et al., 2006). No entanto, não houve necessidade de tratamentos prégerminativos para $B$. cheilantha, a qual obteve elevada taxa de germinação em todos os grupos. Vale ressaltar que quando as sementes de $B$. cheilantha e $A$. polyphylla são atacadas por insetos, é possível que esses danifiquem o embrião, resultando na queda da taxa de germinação (Copeland \& McDonald, 2001).
$\mathrm{O}$ ataque de insetos aos propágulos das espécies da família Fabaceae é comum em ambiente natural e diminui a chance da semente germinar (Janzen, 1971; Sari et al., 2002). Embora algumas sementes atacadas tenham germinado, os insetos encontrados parecem ser efetivos predadores das sementes de $B$. cheilantha e A. polyphylla, e não agentes escarificadores para proporcionar melhor germinação. Os frutos e as sementes representam uma potencial fonte de energia e alimento para os herbívoros (Hulme, 1998) e são utilizados no ciclo de desenvolvimento de vários insetos (Janzen, 1971; 1975). Comumente, Bruchidae são encontrados predando sementes de Fabaceae e, associado a eles, grandes populações de parasitas (Janzen, 1971; Sari et al., 2002). Os Braconidae são parasitas que em seus estados larvais desenvolvem sobre ou dentro de outros insetos não adultos, geralmente Coleoptera (como o Bruchidae encontrado), Lepidoptera, Diptera e Hemíptera (Borror \& Delong, 1969). Por serem parasitóides generalistas, são também utilizados no controle biológico de insetos-praga (Cirelli \& Penteado-Dias, 2003). Perilampídios são, em sua maioria, hiperparasitoides que atacam pupa de Dípteros e Himenópteros parasitas (como o Braconidae encontrado); além de parasitar Taquinídeos, Bruchideos e Icneumonídeos (Borror \& Delong, 1969). Perilampídios também são considerados raros e indicadores de áreas conservadas (Aguiar, 2001). É possível que a pressão causada por esses insetos predadores às populações de $A$. polyphylla e $B$. cheilantha selecione os indivíduos com alta produção de sementes viáveis, a fim de compensar as sementes atacadas. Além disso, outro fator controlador da predação está na interação encontrada entre os predadores e seus parasitas em seu ambiente natural não impactado, possibilitando o pleno desenvolvimento das populações de $A$. polyphylla e $B$. cheilantha típicas das florestas decíduas (Arruda et al., 2011) e formações da Caatinga (Barbosa, 2003; Queiroz, 2006).

\section{Conclusões}

Os tratamentos pré-germinativos não surtiram efeito na germinação de Anadenanthera colubrina. No entanto, as baixas taxas germinativas apresentadas pela espécie foram atribuídas à infestação por fungos.

Acacia polyphylla e Bauhinia cheilantha apresentaram elevada taxa de germinação nos diferentes grupos avaliados, sendo prejudicadas apenas quando suas sementes são atacadas por insetos. Os insetos encontrados 
são comuns predadores de sementes da família Fabaceae. Embora as espécies analisadas tenham se mostrado independentes de tratamentos pré-germinativos para uma boa germinação, a escarificação mecânica em $A$. polyphylla mostrou-se eficiente, pois potencializou ao máximo a germinação da espécie, além de aumentar a velocidade de germinação.

\section{Agradecimentos}

Os autores agradecem à Fundação de Amparo a Pesquisa do Estado de Minas Gerais (FAPEMIG) pelo financiamento do projeto (DEG 2583/05) e a bolsa de doutorado de M.D.M. Veloso. Os autores agradecem também à UNIMONTES pelo apoio logístico, a Lucimar Araújo e Cleandson Santos pela identificação dos insetos e a Eduardo Prata, Flávio Rodrigues, Lelo Carvalho, Patrícia Povoa, Rúbia Fonseca, Ulisses Moliterno e revisores anônimos pelos importantes comentários ao manuscrito. Y.R.F. Nunes agradece ao CNPq (processo 307039/2013-7) pelo apoio.

\section{Referências}

AGUIAR, A. P. Manual de informações sobre conservação e vida silvestre de insetos de Mata Atlântica. São Paulo, 2001. 100 p.

ANTUNES, F. Z. Caracterização climática. Informe Agropecuário, Belo Horizonte, n. 181, p. 15-19, 1994.

ARAÚJO NETO, J. C.; AGUIAR, I. B.; FERREIRA, V. M. Efeito da temperatura e da luz na germinação de sementes de Acacia polyphylla DC. Revista Brasileira de Botânica, São Paulo, v. 26, n. 2, p. 249-256, 2003. DOI: 10.1590/S0100-84042003000200013

ARAÚJO NETO, J. C.; AGUIAR, I. B.; FERREIRA, V. M.; RODRIGUES, T. J. D. Armazenamento e requerimento fotoblástico de sementes de Acacia polyphylla DC. Revista Brasileira de Sementes, Pelotas, v. 27, n. 1, p. 115-124, 2005. DOI: 10.1590/ S0101-31222005000100014

ARRUDA, D. M.; BRANDÃO, D. O.; COSTA, F. V.; TOLENTINO, G. S.; BRASIL, R. D.; NETO, D. Â.; NUNES, Y. R. F. Structural aspects and floristic similarity among tropical dry forest fragments with different management histories in Northern Minas Gerais, Brazil. Revista Árvore, Viçosa, MG, v. 35, n. 1, p. 131-142, 2011. DOI: $10.1590 / \mathrm{S} 0100-67622011000100016$

AUGSPURGER, C. K. Una seña para la floración sincrónica. In: LEIGHT, E. G.; RAND, A. S.; WINDSOR, D. M. (Ed.). Ecologia de um bosque tropical: ciclos estacionales y cambios a largo prazo. Balboa: Smithsonian Institution, 1990. p. 219-442.

BARBOSA, D. C. A. Estratégias de germinação e crescimento de espécies lenhosas da caatinga com germinação rápida. In: LEAL, I. R.; TABARELLI, M.; SILVA, J. M. C. (Ed.). Ecologia e conservação da caatinga. Recife: Universidade Federal de Pernambuco, 2003. p. 625-656.
BEWLEY, J. D.; BLACK, M. Physiology and biochemistry of seeds in relation to germination. Berlim: Springer Verlag, 1985. $367 \mathrm{p}$.

BORGES, E. E. L.; RENA, A. B. Germinação de sementes. In: AGUIAR, I. B.; PIÑA-RODRIGUES, F. C. M.; FIGLIOLIA, M. B. Sementes florestais tropicais. Brasília, DF: Associação Brasileira de Tecnologia de Sementes, 1993. p. 83-135.

BORROR, D. J.; DELONG, D. M. Introdução ao estudo dos insetos. São Paulo: E. Blucher, 1969. 653 p.

CARDOSO, V. J. M. Conceito e classificação da dormência em sementes. Oecologia Brasiliensis, Rio de Janeiro, v. 13, p. 619-631, 2009. DOI: $10.4257 /$ oeco.2009.1304.06

CARVALHO, P. E. R. Espécies arbóreas brasileiras. Brasília, DF: Embrapa Informação Tecnológica; Colombo: Embrapa Florestas, 2003. v. 1. 1040 p.

CARVALHO, P. E. R. Espécies arbóreas brasileiras. Brasília, DF: Embrapa Informação Tecnológica; Colombo: Embrapa Florestas, 2008. v. 3. 604 p.

CARVALHO, L. R.; SILVA, E. A. A.; DAVIDE, A. C. Classificação de sementes florestais quanto ao comportamento no armazenamento. Revista Brasileira de Sementes, Pelotas, v. 28, n. 2, p. 15-25, 2006. DOI: $10.1590 / \mathrm{S} 0101-31222006000200003$

CIRELLI, K. R. N.; PENTEADO-DIAS, A. M. Fenologia dos Braconidae (Hymenoptera, Ichneumonoidea) da Área de Proteção Ambiental (APA) de Descalvado, SP. Revista Brasileira de Entomologia, São Paulo, v.47, n. 1, p. 99-105, 2003. DOI: 10.1590/ S0085-56262003000100015

COPELAND, L. O.; MCDONALD, M. B. Principles of seed science and technology. Massachusetts: Kluwer Academic Pucbishers, 2001.

CORTE, V. B.; BORGES, E. E. D. L.; PONTES, C. A.; LEITE, I. T. D. A.; VENTRELLA, M. C.; MATHIAS, A. D. A. Mobilização de reservas durante a germinação das sementes e crescimento das plântulas de Caesalpinia peltophoroides Benth. (LeguminosaeCaesalpinoideae). Revista Árvore, Viçosa, MG, v. 30, n. 6, p. 941949, 2006. DOI: 10.1590/S0100-67622006000600009

DHINGRA, O. D.; MAIA, C. B.; LUSTOSA, D. C.; MESQUITA, J. B. Seedborne pathogenic fungi that affect seedling quality of red angico (Anadenanthera macrocarpa) trees in Brazil. Journal of Phytopathology, Berlin, v. 150, n. 8-9, p. 451-455, 2002. DOI: 10.1046/j.1439-0434.2002.00777.x

FACHINELLO, J. C.; NACHTIGAL, J. C.; HOFFMANN, A. Propagação por sementes. In: FACHINELLO, J. C.; HOFFMANN, A.; NACHTIGAL, J. C. (Ed.). Propagação de plantas frutíferas. Brasília, DF: Embrapa Informação Tecnológica. 2005. p. 57-67.

FELFILI, J. M.; SILVA JUNIOR, M. C.; DIAS, B. J.; REZENDE, A.V. Estudo fenológico de Stryphnodendron adstringens (Mart.) Coville no cerrado sensu stricto da Fazenda Água Limpa no Distrito Federal, Brasil. Revista Brasileira de Botânica, São Paulo, v. 22, n. 1, p. 83-90, 1999. DOI: 10.1590/S0100-84041999000100011

FIGLIOLIA, M. B.; AGUIAR, I. B.; SILVA A. Germinação de sementes de três espécies arbóreas brasileiras. Revista do Instituto Florestal, São Paulo, v. 21, p. 107-115, 2009. 
GARCIA, L. C.; NOGUEIRA, A. C.; ABREU, D. C. A. Influência do envelhecimento acelerado no vigor de sementes de Anadenanthera colubrina (Vellozo) Brenan - Mimosaceae. Ciência Florestal, Santa Maria, RS, v.14, p. 85-90, 2004.

GARWOOD, N. C. Seed germination in a seasonal tropical forest in Panama: a community study. Ecological monographs, Washington, DC, v. 53, n. 2, p. 159-181, 1983. DOI: 10.2307/1942493

GIVELBERG, A.; HOROWITZ, M.; POLJAKOFF-MAYBER, A. Germination behaviour of Solanum nigrum seeds. Journal of Experimental Botany, Oxford, v. 35, p.588-598, 1984. DOI: $10.1093 / \mathrm{jxb} / 35.4 .588$

HAMMER, Ø.; HARPER, D. A. T.; RYAN, P. D. PAST: paleontologycal statistics software package for education and data analysis. Palaeontologia Electronica, v. 4, p. 1-9, 2001.

HULME, P. E. Post-dispersal seed predation and seed bank persistence. Seed Science Research, Durham, v. 8, n. 4, p. 513-519, 1998. DOI: $10.1017 / \mathrm{S} 0960258500004487$

JANZEN, D. H. Seed predation by animals. Annual Review of Ecology and Systematics, v. 2, p. 465-492, 1971. DOI: 10.1146/ annurev.es.02.110171.002341

JANZEN, D. H. Intra and interhabitat variations in Guazuma ulmifolia (Sterculiaceae) seed predation by Amblycerus cistelinus (Bruchidae) in Costa Rica. Ecology, Tempe, v. 56, n. 4, p. 1009-1013, 1975. DOI: $10.2307 / 1936314$

JANZEN, D. Tropical dry forests. The most endangered major tropical ecosystem. In: WILSON, E. O. (Ed.). Biodiversity. Washington, DC: National Academy of Sciences/Smithsonian Institution, 1988. p. 130-137.

LABOURIAU, L. G. A. A germinação das sementes. Washington, DC: Secretaria Geral da Organização dos Estados Americanos. 1983, 179 p.

LIMA, V. V. F.; VIEIRA, D. L. M.; SEVILHA, A. C.; SALOMÃO, A. N. Germinação de espécies arbóreas de floresta estacional decidual do vale do rio Paranã em Goiás após três tipos de armazenamento por até 15 meses. Biota Neotropica, Campinas, v. 8, n. 3, p. 89-97, 2008. DOI: $10.1590 / \mathrm{S} 1676-06032008000300008$

LOPES, J. C.; DIAS, P. C.; MACEDO, C. M. P. Tratamentos para acelerar a germinação e reduzir a deterioração das sementes de Ormosia nitida vog. Revista Árvore, Viçosa, MG, v. 30, n. 2, p. 171-177. 2006. DOI: 10.1590/S0100-67622006000200003

LORENZI, H. Árvores brasileiras. São Paulo: Plantarum. 1992. v. $1.384 \mathrm{p}$.

LUZ, G. R.; NUNES, Y. R. F. Seed germination of arboreal shrub species with different dispersal mechanisms in a Brazilian Tropical Dry Forest. In: SANCHEZ-AZOFEIFA, A.; POWERS, J. S.; FERNANDES, G. W.; QUESADA, M. (Ed.). Tropical Dry Forests in the Americas: ecology, conservation, and management. Boca Raton: CRC Press, 2013. p. 286-303.

MAGUIRE, J. D. Speed of germination-aid in selection and evaluation for seedlings emergence and vigor. Crop Science, Madson, v. 2, n. 2, p. 176-177, 1962. DOI: $10.2135 /$ cropsci1962.0 011183X000200020033x
MATHEUS, M. T.; LOPES, J. C. Morfologia de frutos, sementes e plântulas e germinação de Erythrina variegata L. Revista Brasileira de Sementes, Londrina, v. 29, n. 3, p. 08-17. 2007. DOI: 10.1590/ S0101-31222007000300002

MELO, R. R.; FERREIRA A. G.; RODOLFO JUNIOR, F. Efeito de diferentes substratos na germinação de sementes de angico (Anadenanthera colubrina (Vell.) Brenan) em condições de laboratório. Revista Científica Eletrônica de Engenharia Florestal, Garça, v. 5, 2005.

MELO-PINNA, G. F. A.; NEIVA, M. S. M.; BARBOSA, D. C. A. Estrutura do tegumento seminal de quatro espécies de Leguminosae (Caesalpinioideae), ocorrentes numa área de caatinga (PE - Brasil). Revista Brasileira de Botânica, São Paulo, v. 22, n. 3, p. 375-379, 1999. DOI: 10.1590/S0100-84041999000300004

NUNES, Y. R. F.; FAGUNDES, M.; SANTOS, M. R.; BRAGA, R. F.; GONZAGA, A. P. D. Germinação de sementes de Guazuma ulmifolia Lam. (Malvaceae) e Heteropterys byrsonimifolia A. Juss (Malpighiaceae) sob diferentes tratamentos de escarificação tegumentar. Unimontes Cientifica, Montes Claros, v. 8, n. 1, p. 43-52, 2006.

NUNES, Y. R. F.; FAGUNDES, M.; ALMEIDA, H. S.; VELOSO, M. D. M. Aspectos ecológicos da aroeira (Myracrodruon urundeuva Allemão- Anacardiaceae): fenologia e germinação de sementes. Revista Árvore, Viçosa, MG, v. 32, n. 2, p. 233-243, 2008. DOI: 10.1590/S0100-67622008000200006

OLIVEIRA, D. A.; NUNES, Y. R. F.; ROCHA, E. A.; BRAGA, R. F.; PIMENTA, M. A. S.; VELOSO, M. D. M. Potencial germinativo de sementes de fava-d'anta (Dimorphandra mollis Benth. - Fabaceae: Mimosoideae) sob diferentes procedências, data de coleta e tratamentos de escarificação. Revista Árvore, Viçosa, MG, v. 32, n. 6, p. 1001-1009, 2008. DOI: 10.1590/S0100-67622008000600005

OLIVEIRA-FILHO, A. T.; JARENKOW, J. A.; RODAL, M. J. N. Floristic relationships of seasonally dry forests of eastern South America based on tree species distribution patterns. In: PENNINGTON, R. T.; LEWIS, G. P.; RATTER, J. A. (Ed.). Neotropical savannas and dry forests: plant diversity, biogeography, and conservation. Oxford: Taylor \& Francis CRC Press. 2006. p. 59-192.

PEREIRA, M. S. Manual técnico: conhecendo e produzindo sementes e mudas da caatinga. Fortaleza: Associação Caatinga, 2011. $86 \mathrm{p}$.

POPINIGIS, F. Fisiologia de sementes. Brasília, DF: AGIPLAN, 1985. 289 p.

QUEIROZ, L. P. The Brazilian Caatinga: phytogeographical pattern inferred from distribution data of the Leguminosae. In: PENNINGTON, R. T.; LEWIS, G. P.; RATTER, J. A. (Ed.). Neotropical Savannas and dry forests: plant diversity, biogeography, and conservation. Oxford: Taylor \& Francis CRC Press. 2006. p. 113-149.

RODRIGUES, E. H. A.; AGUIAR, I. B.; SADER, R. Quebra de dormência de sementes de três espécies do gênero Cassia. Revista Brasileira de Sementes, Brasília, DF, v. 12, p. 17-25, 1990. 
RODRIGUES, A. C. C.; OSUNA, J. T. A.; QUEIROZ, S. R. O. D.; RIOS, A. P. S. Efeito do substrato e luminosidade na germinação de Anadenanthera colubrina (Fabaceae, Mimosoideae). Revista Árvore, Viçosa, MG, v. 31, n. 2, p. 187-193, 2007. DOI: 10.1590/ S0100-67622007000200001

SÁNCHEZ-AZOFEIFA, G. A.; QUESADA, M.; PRODRÍGUEZ, J. P.; NASSAR, J. M.; STONER, K. E.; CASTILLO, A.; GARVIN, T.; ZENT, E. L.; CALVO-ALVARADO, J. C.; KALACSKA, M. E. R.; FAJARDO, L.; GAMON, J. A.; CUEVAS-REYES, P. Research priorities for Neotropical dry forests. Biotropica, Washington, US, v. 37, n. 4, p. 477-485, 2005. DOI: 10.1046/j.0950091x.2001.00153.x-i1
SARI, L. T.; RIBEIRO-COSTA, C. S.; MEDEIROS, A. C. S. Insects associated with seeds of Lonchocarpus muehlbergianus hassl. (Fabaceae) in Três Barras, Paraná, Brazil. Neotropical Entomology, Londrina, v. 31, n. 3, p. 483-486, 2002. DOI: 10.1590/S1519566X2002000300023

SILVA, A.; FIGLIOLIA, M. B.; AGUIAR, I. B. Germinação de sementes de Acacia polyphylla DC. (monjoleiro) e de Aspidosperma ramiflorum Müll. Arg.(guatambu). Floresta, Curitiba, v. 37, n. 3, p. 353-361, 2007. DOI: 10.5380/rf.v37i3.9931

ZAIDAN, L. B. P.; BARBEDO, C. J. Quebra de Dormência. In: FERREIRA, A. G.; BORGUETTI, F. (Ed.). Germinação: do básico ao aplicado. Porto Alegre: Artmed, 2004. p. 135-146. 